\title{
OPEN Conformal smoothing of mid- spatial frequency surface error for nano-accuracy Continuous Phase Plates (CPP)
}

\author{
Ci Song ${ }^{1,2,3}$, Wanli Zhang ${ }^{1,2,3 *}$, Feng Shi ${ }^{1,2,3}$, Zhifan $\operatorname{Lin}^{1,2,3}$ \& Xuqing $\mathrm{Nie}^{1,2,3}$
}

This paper presented a conformal smoothing theory, and smoothing capability evaluation was established on the proposed theory. According to pressure distribution model, processing parameters have been optimized and the CPP sample with a size of $340 \times 340 \mathrm{~mm}$ was applied in conformal smoothing. The middle spatial frequency was effectively corrected with the total polishing time of $750 \mathrm{~min}$, and energy was constringed 32.2 times (improved from $57.68 \mathrm{~nm}^{2} \cdot \mathrm{mm}$ to $1.79 \mathrm{~nm}^{2} \cdot \mathrm{mm}$ ). Meanwhile, surface roughness RMS (root mean square) maintained at the same scale (changed from $265.4 \mathrm{~nm}$ to $265.2 \mathrm{~nm}$ ). Parametric conformal smoothing was proven to be an effective method to control the middle spatial frequency error of CPPs.

Continuous phase plate (CPP) with continuously varying topographical microstructures is a typical diffractive optical element, and it has wide spread applications in modern optics field of beam shaping, compensation and modulation ${ }^{1,2}$ CPPs surface have spatial microstructure, fractal surface, and large grads, which are different from traditional optics and common microstructure array component. The phase unit characteristic size, PV (peak-to-valley), and surface grads of typical CPPs are $5 \sim 50 \mathrm{~mm}, 1 \sim 10 \mu \mathrm{m}$, and $1 \sim 5 \mu \mathrm{m} / \mathrm{cm}$ respectively. The requirements on gradient and spatial frequency make it difficult to fabricate.

Magnetorheological finishing (MRF), an advanced optical finishing process combining interferometry, precision equipment, and computer control, has been an effective method to process $\mathrm{CPPs}^{3-6}$. During the polishing process, dwell time is accurately controlled to get CPPs structure with nanometer accuracy. However, MRF has a few matters lead to mid-to-high spatial frequency error: ${ }^{7-10}$ (1) removal function fluctuation including removal function size or removal efficiency fluctuation. (2) CCOS (computer controlled optical surfacing) convolutional residual error produced by manufacturing route. The mid-to-high spatial frequency error on CPPs surface leads to serious problems such as grating effect and far-field light intensity modulation.

Limited by the size of polishing tool in MRF, IBE and atmospheric pressure plasma processing (APPP) have been chosen to fabricate $C P P s^{11,12}$. For IBE, fabrication route and removal function fluctuation restrict the improvement of surface precision, while post-treatment need be applied to remove residues in APPP.

Existing smoothing theories and technologies have effectively controlled mid-to-high spatial frequency error. Researchers have manufactured many aspheric surfaces using flexible plates ${ }^{13-16}$. Mehta Firstly derived pressure distribution model of flexible smoothing plate in aspheric surface manufacture based on Bridge Model. Dae Wook Kim developed the smoothing model of RC plate and then compared smoothing factors of pitch tools and RC plates ${ }^{17}$. Walker proposed pseudo-random tool paths for CNC sub-aperture polishing ${ }^{18}$. Yifan Dai proposed local random processing path based on maximum entropy method ${ }^{19}$. Hon-Yuen Tam proposed Peano-like paths for sub-aperture polishing of optical aspherical surfaces ${ }^{20}$. All of the techniques were confirmed that had good smoothing effects, while they could not be used to fabricate CPPs because of the change in microstructure.

Most of existing smoothing theories concentrate on a single frequency, while the relationships of low-spatial frequency surface and middle spatial frequency could not be described simultaneously. Hence, conformal smoothing should be developed, factors to quantitatively evaluate smoothing and conformal capability should

${ }^{1}$ College of Intelligent Science and Technology, National University of Defense Technology, Changsha, 410073, China. ${ }^{2}$ Hunan Key Laboratory of Ultra-Precision Machining Technology, Changsha, 410073, China. ${ }^{3}$ Laboratory of Science and Technology on Integrated Logistics Support, National University of Defense Technology, Changsha, 410073, China. *email: 564841540@qq.com 


\begin{tabular}{|l|l|l|}
\hline Material & $\mathbf{E}(\mathbf{P a})$ & $\boldsymbol{\nu}$ \\
\hline Steel & $2.09 \mathrm{E}+11$ & 0.269 \\
\hline Aluminum & $6.90 \mathrm{E}+10$ & 0.33 \\
\hline ABS & $2.00 \mathrm{E}+09$ & 0.39 \\
\hline Silica gel & $1.00 \mathrm{E}+05$ & 0.35 \\
\hline
\end{tabular}

Table 1. Physical properties of four materials.

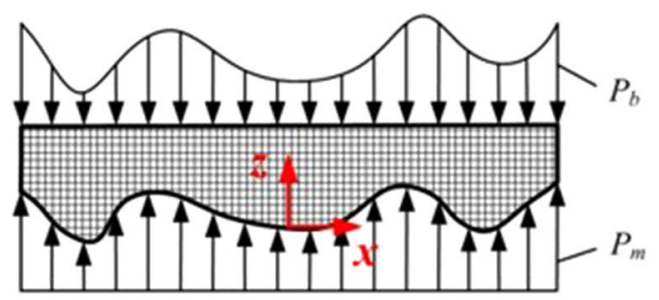

(a)

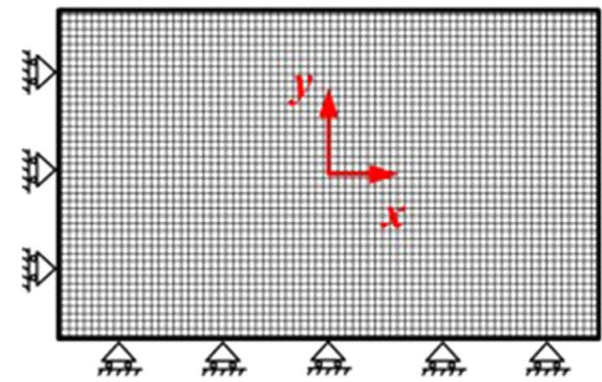

(b)

Figure 1. Meshed Finite Element Method model of the polishing pad: (a) Front view (b) top view.

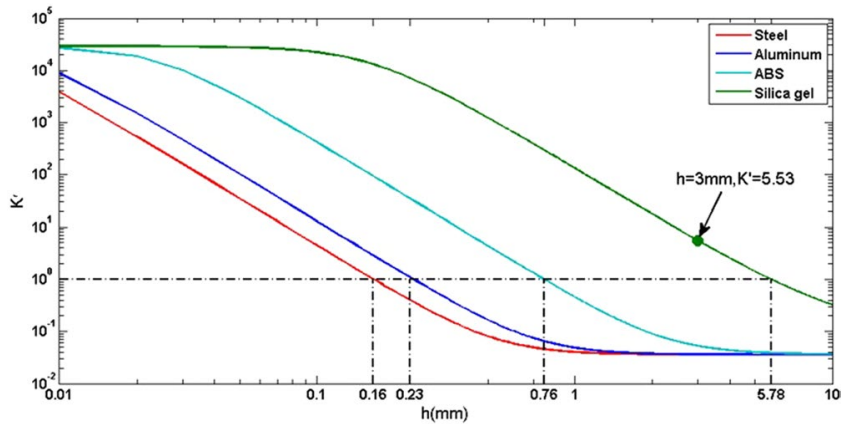

Figure 2. Simulation of the smoothing ratio $K^{\prime}$ with different materials and thickness.

be completed. Conformal smoothing aims to smooth mid-to-high spatial frequency of millimeter level and keep specific low-spatial frequency surface precision. Conformal SP should be optimized.

This paper focuses on theoretic analysis of SP, optimization of conformal smoothing tool, and engineering applications of conformal smoothing. Firstly, the conception of smoothing ratio was proposed to characterize the effect of smoothing and conformability based on pressure distribution model. Then, the smoothing tool was optimized by theoretically analyzing, simulating, and experimentally verifying. Finally, the optimized tool was applied in the smoothing of $340 \mathrm{~mm} \times 340 \mathrm{~mm}$ CPP, where mid-to-high spatial frequency errors were effectively controlled.

\section{Theory of Conformal Smoothing}

Pressure distribution model. To calculate the pressure distribution, a generalized model is established in this research. In this model, the contact between polishing pad and component is considered to be elastic and the component is assumed to be rigid. Effects friction and others are ignored, in order to simplify the model ${ }^{21,22}$.

As shown in Fig. 1, $\mathrm{P}_{\mathrm{b}}$ is the force exerted by polishing pad. $\mathrm{P}_{\mathrm{m}}$ is the supporting force.

According to laws of minimum potential energy and Hooke's, the governing matrix equation of hexahedral elements separated from pressure distribution model could be defined as follows:

$$
\frac{\partial G_{0}}{\partial C_{0}}=F_{0}=K_{0} C_{0}, \quad K_{0}=\int_{v} B_{0}^{T} D_{0} B_{0} d v
$$

$\mathrm{F}_{0}$ is the nodal force vector. $\mathrm{K}_{0}$ is the element stiffness matrix. $\mathrm{C}_{0}$ is the nodal displacement vector. $\mathrm{B}_{0}$ is the strain-displacement matrix. $\mathrm{D}_{0}$ is the elastic stress-strain relation matrix.

Both matrices mentioned in last equation are related to properties of polishing tool and mid-spatial frequency error $(\mathrm{MSFR})^{23}$. 


\begin{tabular}{|l|l|}
\hline Material of base & Silica gel \\
\hline Thickness of base & $3 \mathrm{~mm}$ \\
\hline Thickness of pitch & $2 \mathrm{~mm}$ \\
\hline Diameter of tool & $15 \mathrm{~mm}$ \\
\hline Pressure of smoothing & $0.05 \mathrm{MPa}$ \\
\hline Revolution speed of tool & $100 \mathrm{rpm}$ \\
\hline Spin speed of tool & $105 \mathrm{rpm}$ \\
\hline Smoothing tool-path & raster \\
\hline Smoothing time in a run & $10 \mathrm{~min}$ \\
\hline Polishing abrasive & $\mathrm{CeO}_{2}$ \\
\hline Abrasive size & $1 \mu \mathrm{m}$ \\
\hline
\end{tabular}

Table 2. Smoothing parameters.

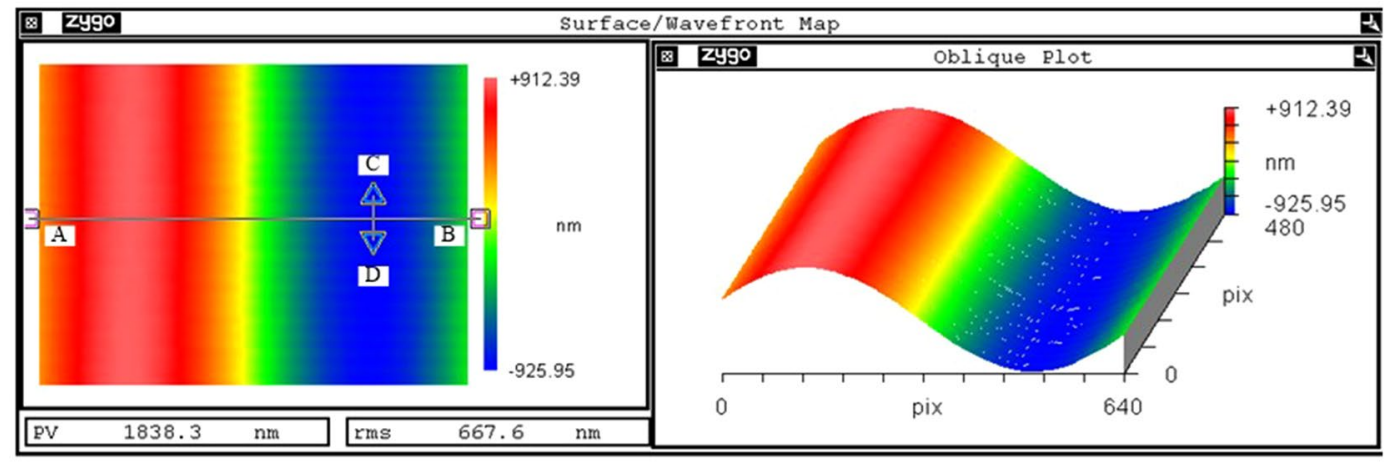

(a)

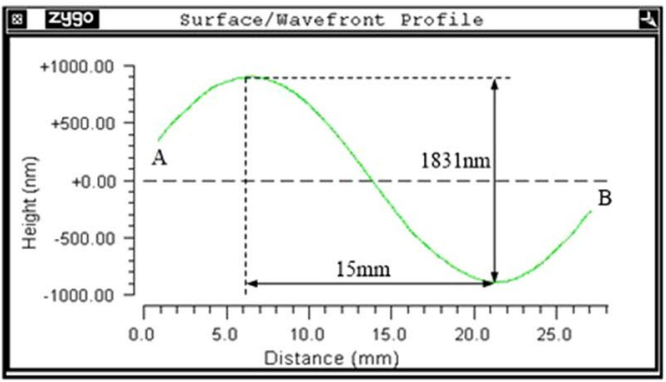

(b)

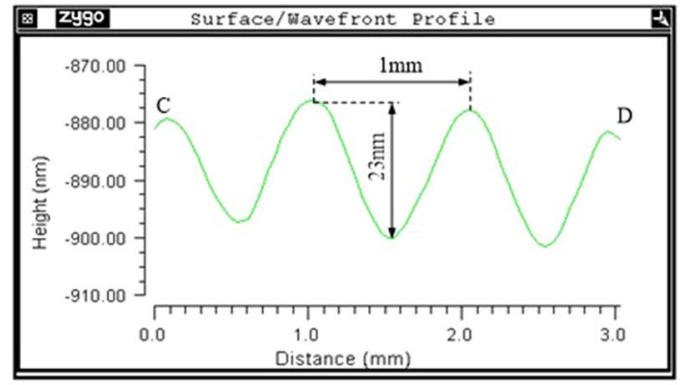

(c)

Figure 3. Sine surface error manufactured by MRF. (a) Local surface map distribution, (b) sectional diagram in the horizon direction (c) sectional diagram in the vertical direction.

Then, integrated every vector in Eq. (1) and the whole matrix could be defined as follows:

$$
F_{\text {all }}=K_{\text {all }} \cdot C_{\text {all }}
$$

Meanwhile, the boundary conditions could be described as following equations set before solving the model.

$$
\left\{\begin{array}{l}
C_{m}=\text { error } \\
\frac{\partial\left(C_{\mathrm{b}}\right)}{\partial z}=0 \\
u_{x=0}=0, \quad v_{y=0}=0 \\
\sum F_{b}=-\sum F_{m}, \quad \sum F_{b}=f \cdot \frac{S_{u}}{S}
\end{array}\right.
$$

$\mathrm{C}_{\mathrm{m}}$ and $\mathrm{C}_{\mathrm{b}}$ are the displacements of lower and upper surface. error is the MSFR on the mirror. $\mathrm{u}$ and $\mathrm{v}$ are the displacements in the $\mathrm{x}$ and $\mathrm{y}$ direction. $\mathrm{F}_{\mathrm{b}}$ is the nodal force of upper surface. $\mathrm{F}_{\mathrm{m}}$ is the nodal force of lower surface. $f$ is the whole force applied on the lap. $S_{\mathrm{u}}$ is the upper surface area. $S$ is the whole area of the pad.

In the model, the pressure distribution on upper and lower surface can be attained from $\mathrm{F}_{\mathrm{b}}$ and $\mathrm{F}_{\mathrm{m}}$. Further, the material removal rate of MSFR can be calculated by Preston's equation ${ }^{24}$. 
(a)

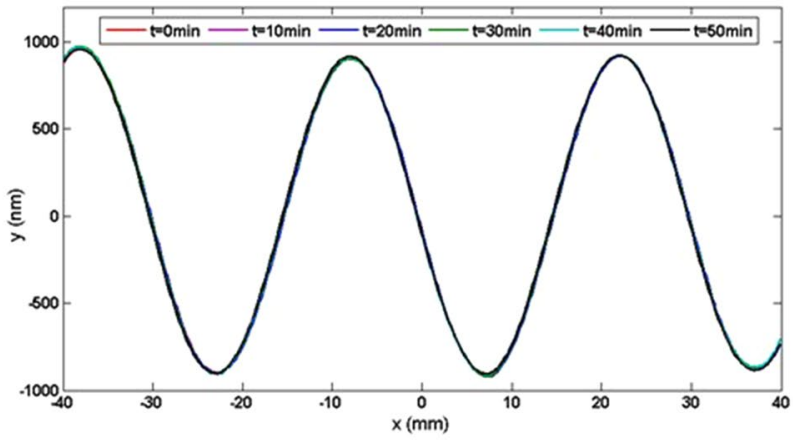

(b)

(c)

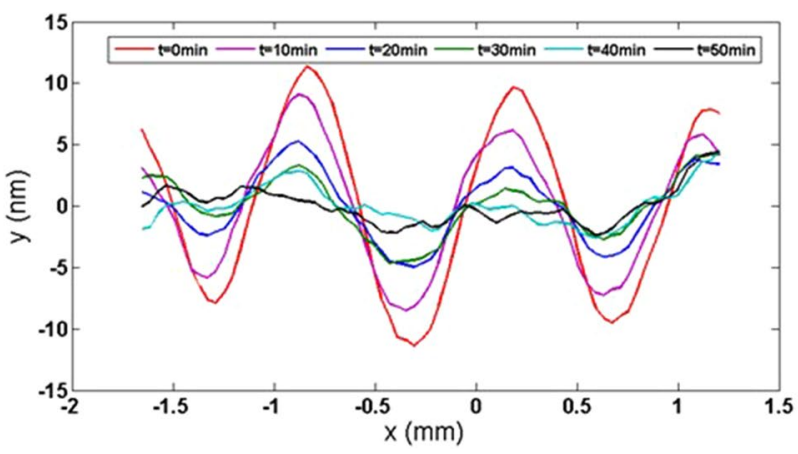

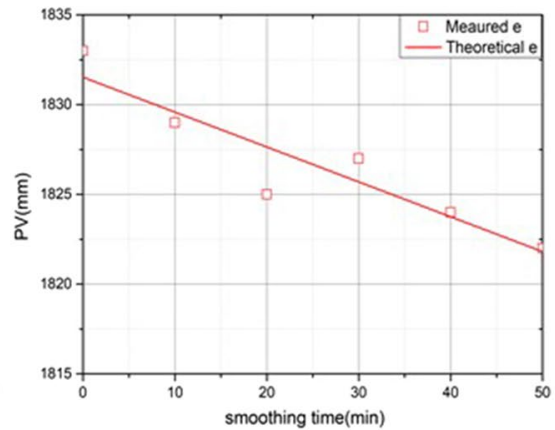

(d)

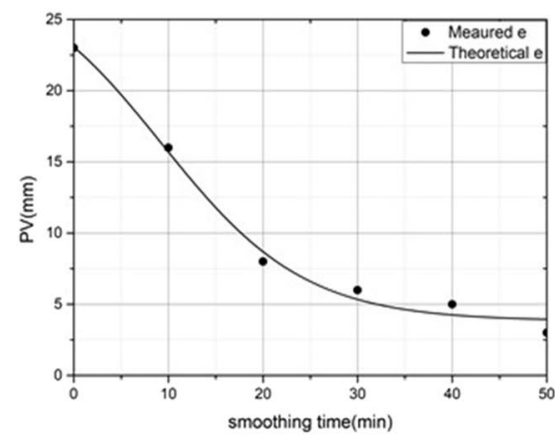

Figure 4. Convergence process with two different periodic surface errors. (a) Sectional diagram of $30 \mathrm{~mm}$ periodic surface error; (b) PV convergence curve of $30 \mathrm{~mm}$ periodic surface error; (c) sectional diagram of $1 \mathrm{~mm}$ periodic surface error; (d) PV convergence curve of $1 \mathrm{~mm}$ periodic surface error.

Smoothing ratio $K^{\prime}$. Smoothing ratio $K^{\prime}$ represents the efficiency of PV convergence of different frequency errors. According to Preston's equation it could be defined as follows:

$$
K^{\prime}=\frac{\mathrm{d}\left(p v_{f 1}\right)}{\mathrm{d}\left(p v_{f 2}\right)}=\frac{\Delta p_{f 1}}{\Delta p_{f 2}}
$$

$\Delta P_{f 1}$ is the pressure of error frequency $f_{1} . \Delta P_{f 2}$ is the pressure of error frequency $f_{2}$. Based on Eq. (4), the following conclusions can be obtained.

(1) $K^{\prime}$ can describe the smoothing effects of different spatial frequency surface errors with variant smoothing tool.

(2) The larger the smoothing ratio $K^{\prime}$ is, the better the smoothing ability of mid-to-high spatial frequency surface error, and the better the conformal ability of low-spatial frequency surface error will be.

(3) Based on pressure distribution model, the smoothing ratio $K^{\prime}$ would change along with parameters in smoothing process.

\section{Conformal Smoothing Validation}

Optimization of smoothing tool. There are a lot of parameters affecting the smoothing ratio including the periodicity of surface error $a$, the elastic modulus $E$, the Poisson's ratio $v$, the thickness of base $h$, and the amplitude of surface error PV. The periodicity of surface error $a$ and the amplitude of surface error PV are mainly dominated by the surface error, and the other parameters are dominated by the material and thickness of polishing tool. In the optimized process of smoothing tool, the material and thickness of base will be optimized to maximize the smoothing ratio in a reasonable range. In smoothing process, the size of smoothing tool is one half of low-spatial frequency error periodicity and the convergence in full bands of errors is achieved ${ }^{25}$.

To optimize the material of base, four different materials (steel, aluminum, ABS resin, silica gel) are compared. The elastic modulus $E$ and Poisson's ratio $v$ are both listed in Table 1 . To understand the SP, the smoothing ratio of the $30 \mathrm{~mm}$ periodic surface error to $1 \mathrm{~mm}$ tool-path with different base thickness are simulated respectively, as shown in Figs. 1 and 2. In the simulation process, the compression rigidity of pitch $K c=4.4 \mathrm{e}^{9} \mathrm{~N} / \mathrm{m}^{326}$.

In Fig. 2, we can find that the smoothing ratio $K^{\prime}$ reduces rapidly when the thickness of base increases, and it tends to be the same when the base thickness further increase. Also, when the elastic modulus $E$ decreases, the 


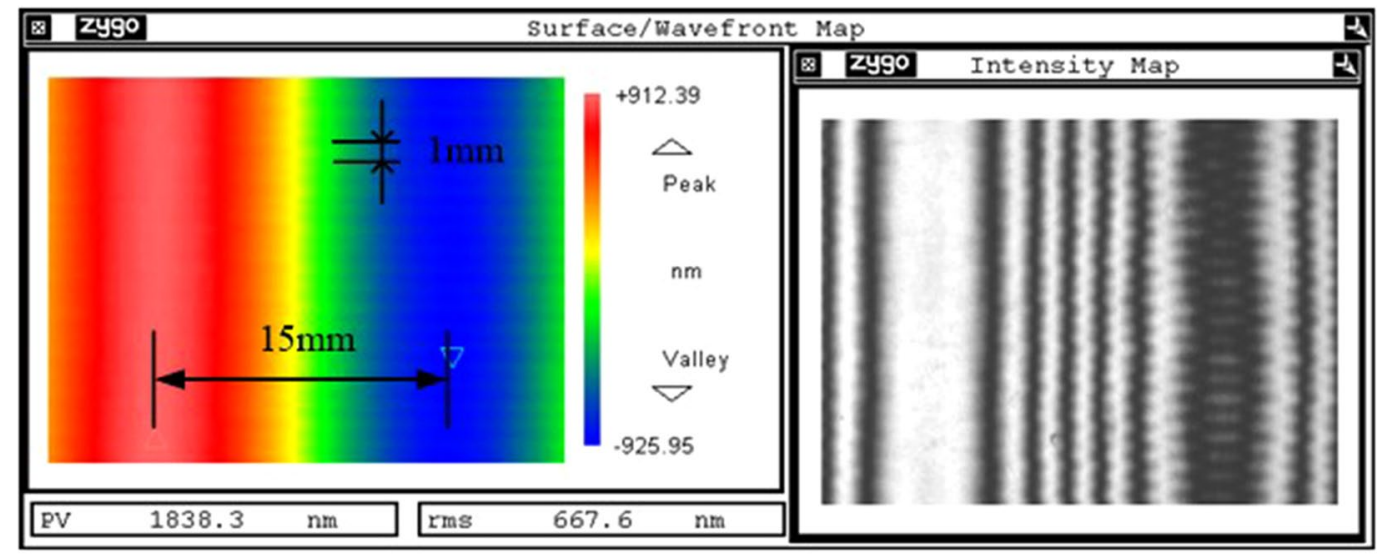

(a)

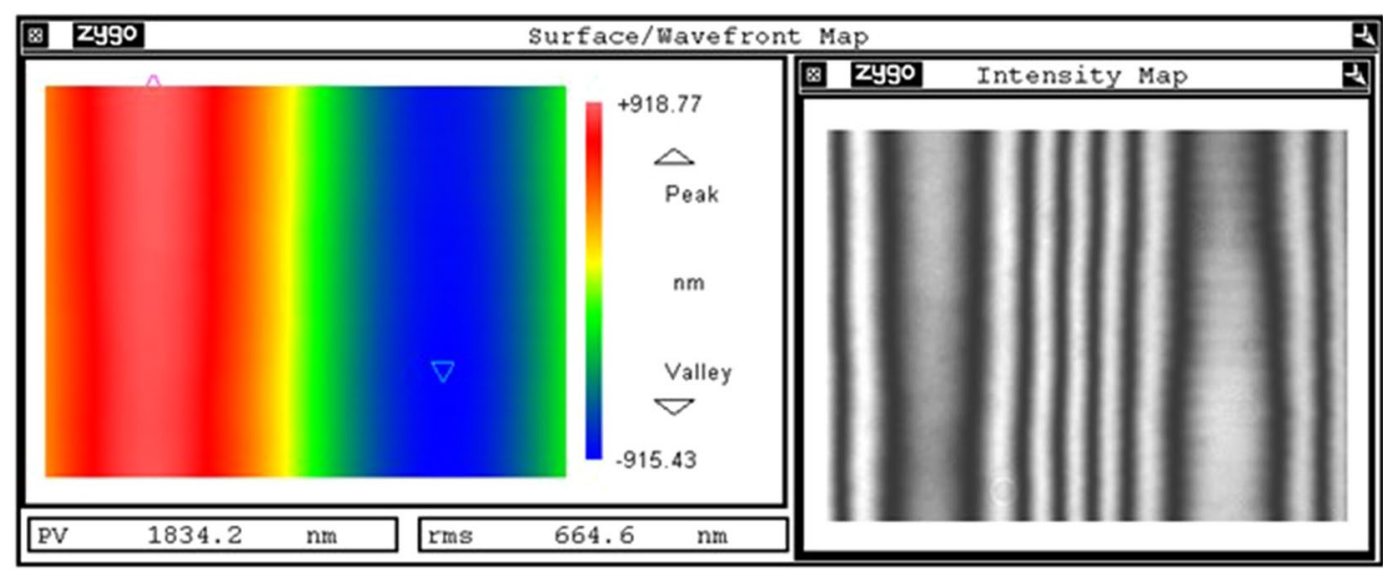

(b)

Figure 5. Smooth results (a) surface map distribution before the SP; (b) surface map distribution after 5 smoothing run.

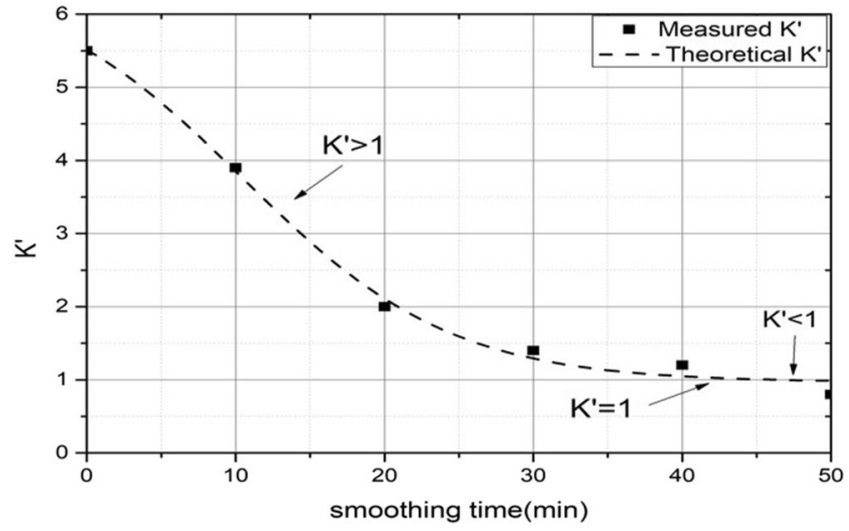

Figure 6. The smoothing ratio curve in the SP.

smoothing ratio increases rapidly. To obtain a large smoothing ratio, the soft base material is recommended. As limited by the structure of the smoothing tool, it is difficult to make the thickness of base less than $3 \mathrm{~mm}$. Therefore, the material and thickness of base are chosen as Silica gel and $3 \mathrm{~mm}$, respectively. The simulation results show that the smoothing ratio is 5.53 when $3 \mathrm{~mm}$ Silica gel is used as the smoothing tool. 


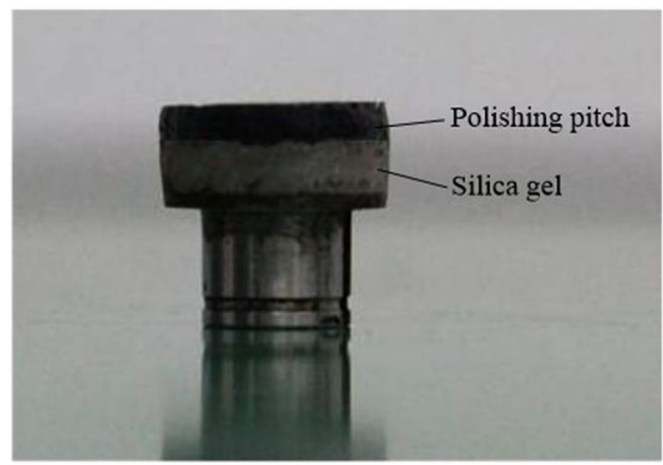

(a)

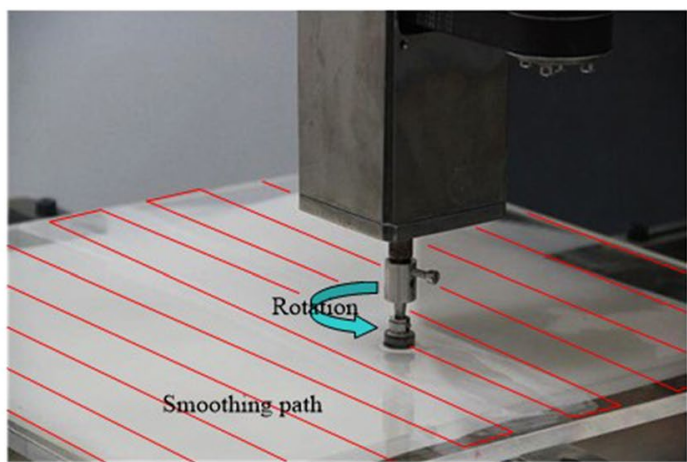

(b)

Figure 7. CPP SP. (a) Smoothing tool photo (b) smoothing tool-path photo.

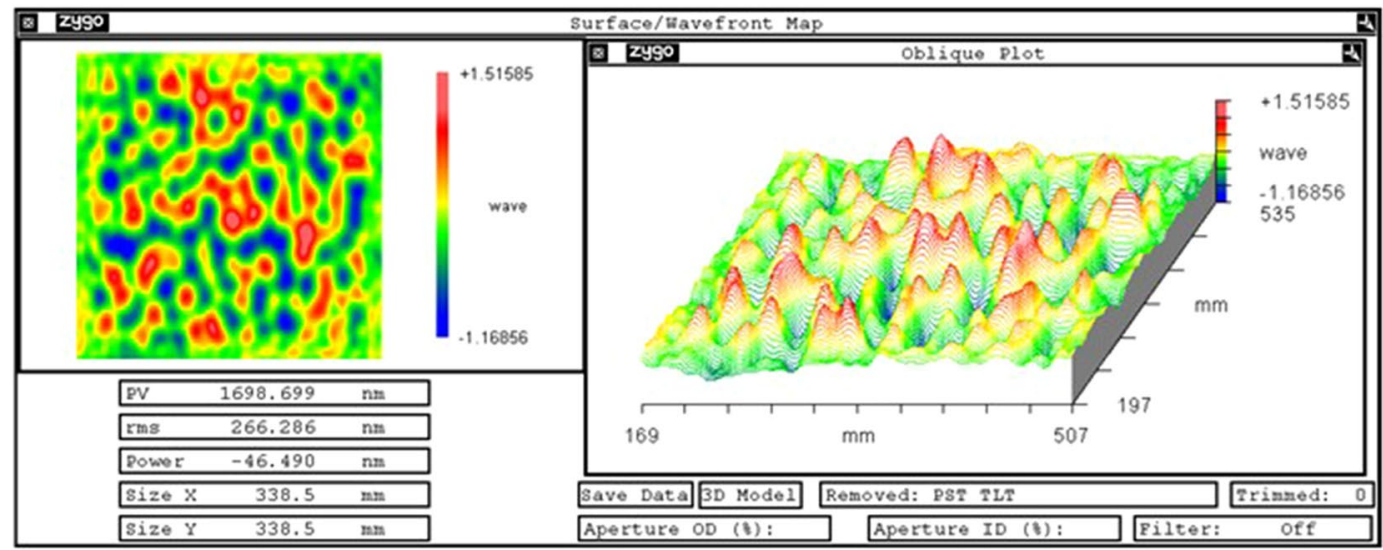

(a)

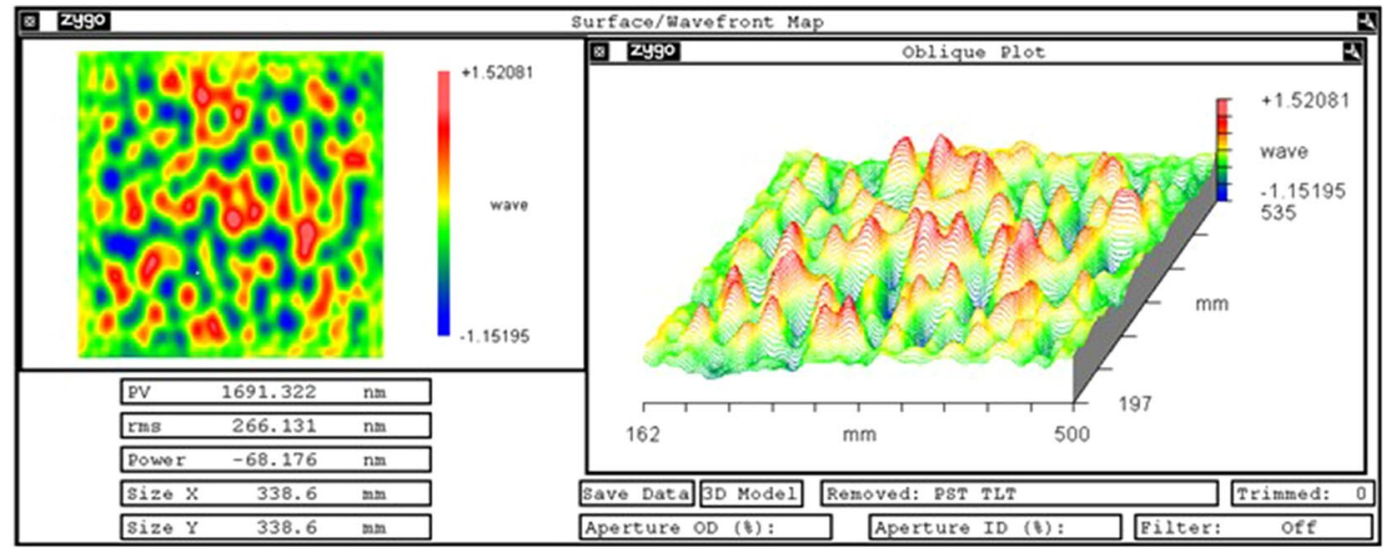

(b)

Figure 8. CCP smoothing results. (a) surface map before smoothing; (b) surface map after smoothing.

Smoothing experiments. To validate the conformal smoothing results, different spatial frequency surface error is used to smooth. As the typical spatial period and amplitude of surface error is $1 \sim 50 \mathrm{~mm}$ and $0.1 \sim 5 \mu \mathrm{m}$, we choose two types of sine spatial frequency surface error to perform the smoothing experiments. One type of sine surface error is $30 \mathrm{~mm}$ periodic and $1831 \mathrm{~nm} \mathrm{PV}$, and another type of sine surface error is $1 \mathrm{~mm}$ periodic and $23 \mathrm{~nm}$ PV. The two types of sine surface error which both manufactured by MRF polishing tool are on the same workpiece but with different direction (one is on $\mathrm{x}$ direction, another is on y direction), as shown in Fig. 3(a).

The smoothing parameters are all listed in Table 2. The time of one smoothing run is $10 \mathrm{~min}$, and the PV of two types of surface error are measured and depicted in every smoothing run, as shown in Fig. 4 


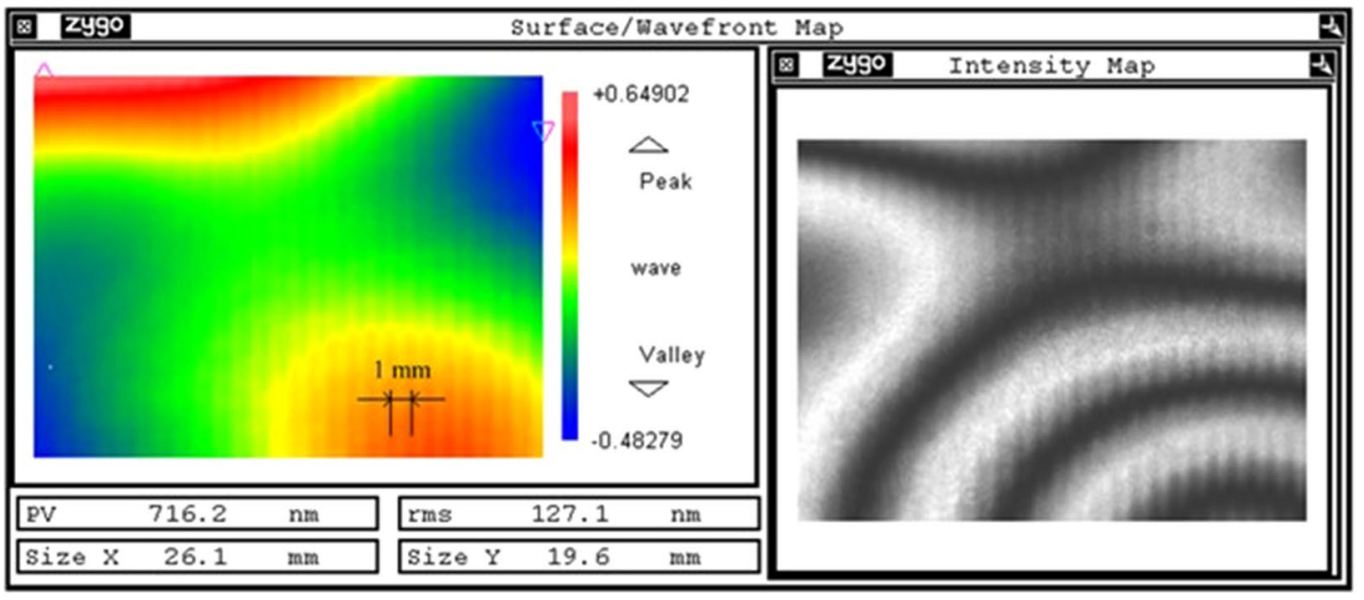

(a)

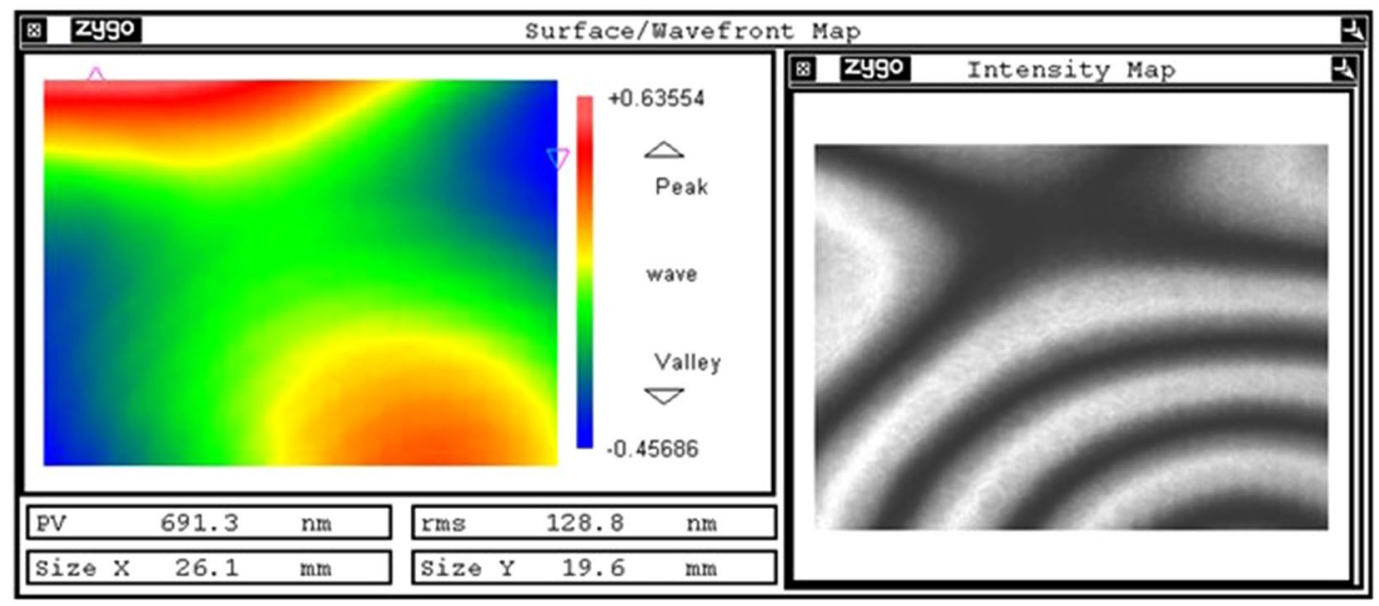

(b)

Figure 9. Sub-aperture test results. (a) Sub-aperture surface map before smoothing; (b) sub-aperture surface map after smoothing.

Although the amplitude of $30 \mathrm{~mm}$ periodic surface error is higher than the amplitude of $1 \mathrm{~mm}$ periodic surface error, the PV convergence ratio of $1 \mathrm{~mm}$ periodic surface error is the faster one. It is observed that the $1 \mathrm{~mm}$ periodic surface error can be smooth in 5 smooth run process. However, the $30 \mathrm{~mm}$ periodic surface error almost the same in the 5 smooth run process and its PV value changes from $1831.5 \mathrm{~nm}$ to $1822.5 \mathrm{~nm}$. The change of surface feature before and after the SP are shown in Fig. 5. In Fig. 5(a), not only the $30 \mathrm{~mm}$ periodic surface error but also the $1 \mathrm{~mm}$ periodic surface error are both obvious in the interferometry image. In Fig. 5(b), it is found that the $1 \mathrm{~mm}$ periodic surface error almost disappears, but the $30 \mathrm{~mm}$ periodic surface error still exists. This experiment demonstrates the smooth effectivity of mid-to-high spatial frequency surface error. It also demonstrates the conformal ability of low-spatial frequency surface error.

The smoothing ratio is also investigated in every smoothing run process, as shown in Fig. 6. The initial actual smoothing ratio is 5.53 which is almost agree with the theoretical value of 5.51 . The smoothing ratio reduces with the increasing of smoothing times and runs. The final actual smoothing ratio is 0.80 which is also agreement with the theoretical value of 0.91 .

Based on Fig. 6, the conformal SP can be divided into three steps. (1) At the beginning of SP, the smoothing ratio $K^{\prime}>1$. The convergence rate of $1 \mathrm{~mm}$ periodic surface error is faster than the $30 \mathrm{~mm}$ periodic surface error. It means that the smoothing effect of mid-to-high spatial frequency surface error is faster than the low-spatial frequency surface error. The smoothing effect plays a major role in the mid-to-high spatial frequency surface error, and the conformal effect plays a major role in the low-spatial frequency surface error in the first step. (2) When the smoothing ratio $K^{\prime}=1$, there is a balance between smoothing effect and conformal effect. At this theoretical point, the smoothing rate of mid-to-high spatial frequency surface error is theoretically the same as the low-spatial frequency surface error. (3) When the smoothing ratio $K^{\prime}<1$, the smoothing rate of mid-to-high spatial frequency surface error is less than the low-spatial frequency surface error. The remove of low-spatial 


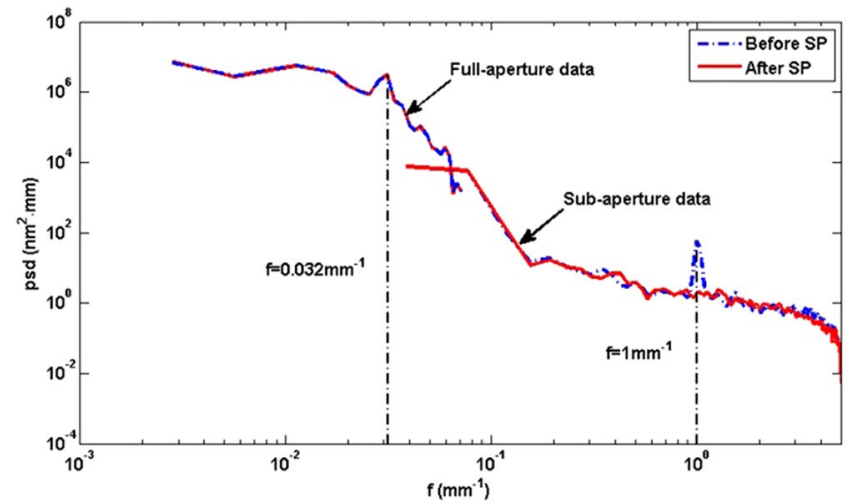

Figure 10. Full-aperture and sub-aperture PSD curve before and after SP.

frequency surface error plays a major role, which the conformal ability will decrease and the surface accuracy tends to be worse in this process.

\section{Application of Conformal Smoothing for CPP}

A $340 \mathrm{~mm} \times 340 \mathrm{~mm}$ fused silica CPP is used to perform the conformal SP, the smoothing tool and parameters are also the same with the above experiments, as listed in Table 2 . The only difference is that the smoothing time of CCP increases to $150 \mathrm{~min}$. The machine tool used to smooth is developed by ourselves. The smoothing tool mainly uses pitch and silica gel, as shown in Fig. 7(a). The smoothing tool-path is raster, as shown in Fig. 7(b), and there are 5 smoothing runs totally.

The full surface map before and after smoothing are both measured by Zygo interferometer VeriFire MST:GPI XP/D,a. Figure 8(a) shows the surface map before smoothing, the PV and RMS is $1698.699 \mathrm{~nm}$ and $266.286 \mathrm{~nm}$, respectively. The PV and RMS is $1691.322 \mathrm{~nm}$ and $266.131 \mathrm{~nm}$ after 5 smoothing runs (750 minutes), as shown in Fig. 8(b).

The CCP is polished by MRF before, and the mesh size is $1 \mathrm{~mm}$. Normally speaking, there will remain $1 \mathrm{~mm}$ periodic residual surface error on the surface. As the aperture of the interferometer is large, the data resolution is not enough to obtain the tool-path. To investigate the residual surface error before and after smoothing, the sub-aperture measurement is used. One sub-aperture can be zoomed largest, then the field range is $26.1 \mathrm{~mm} \times 19.6 \mathrm{~mm}$ and the resolution is $0.0408 \mathrm{~mm} /$ pix. To compare the mid-to-high spatial frequency surface error before and after smoothing, the tested sub-aperture zone should be the same.

Figure 9 shows the same sub-aperture surface interferometry image before and after smoothing. There remain MRF tool-path residuals on the surface before smoothing. The max amplitude of tool-path residuals is almost $11.3 \mathrm{~nm}$, and it reduces to $2.28 \mathrm{~nm}$ after smoothing. There are obvious ripples in the interferometry image before smoothing. However, after smoothing the ripples are difficult to observe on the surface. The results demonstrate that the SP can effectively remove the mid-to-high spatial frequency ripple generated in the MRF process.

To evaluate the SP further, the PSD curve before and after smoothing is calculated, as shown in Fig. 10. The full-aperture PSD mainly focuses on the low-spatial frequency surface error, in which the $30 \mathrm{~mm}$ periodic surface error feature (spatial frequency $\mathrm{f}=0.032 \mathrm{~mm}^{-1}$ ) is almost the same. Besides, all the low-spatial frequency surface error are almost invariant, which demonstrate the conformal ability for low-spatial frequency surface error. The sub-aperture PSD mainly focuses on the mid-to-high spatial frequency surface error, in which the $1 \mathrm{~mm}$ periodic surface error feature (spatial frequency $\mathrm{f}=1 \mathrm{~mm}^{-1}$ ) have been correctted obviously. The energy reduces from $57.68 \mathrm{~nm}^{2} \cdot \mathrm{mm}$ to $1.79 \mathrm{~nm}^{2} \cdot \mathrm{mm}$, it demonstrates the smoothing ability for mid-to-high spatial frequency surface error.

\section{Conclusion}

(1) Based on the pressure distribution model, the smoothing ratio is proposed to describe the convergence rate of different periodic surface errors and evaluate the smoothing and conformal ability.

(2) The smoothing tool is optimized based on the smoothing ratio. The simulation agrees with the smoothing experiments, which demonstrates the correctness of the smoothing ratio.

(3) The optimized smoothing tool and parameters are used to smooth a $340 \mathrm{~mm} \times 340 \mathrm{~mm}$ CPP sample polished by MRF. The PSD curve indicates that the low-spatial frequency surface error can be conformal and the tool-path can be eliminated effectively, the energy reduces from $57.68 \mathrm{~nm}^{2} \cdot \mathrm{mm}$ to $1.79 \mathrm{~nm}^{2} \cdot \mathrm{mm}$ and the surface accuracy RMS only changes from $266.3 \mathrm{~nm}$ to $266.1 \mathrm{~nm}$ in 5 smoothing run process with total $750 \mathrm{~min}$. The CCP application demonstrates that the SP can not only conform the low-spatial surface accuracy but also eliminates the mid-to-high spatial frequency surface error.

\section{Data availability}

All data, models, during the study appear in the submitted article.

Received: 25 June 2019; Accepted: 28 January 2020;

Published online: 13 February 2020 


\title{
References
}

1. Dixit, S. N., Feit, M. D., Perry, M. D. \& Powell, H. T. Designing fully continuous phase screens for tailoring focal-plane irradiance profiles. Opt. Lett. 21, 1715-1717 (1996).

2. Rui, Z. et al. Research of beam conditioning technologies using continuous phase plate, Multi-FM smoothing by spectral dispersion and polarization smoothing. Opt. Lasers Eng. 85, 38-47 (2016).

3. A. Kulawiec, W. Kordonski \& S. Gorodkin "New Approaches to MRF, Imaging and Applied Optics Technical Digest." (2012).

4. Hao, H., Yifan, D., Xiaoqiang, P. \& Jianmin, W. Research on reducing the edge effect in magnetorheological finishing. Appl. Opt. 50, $1220-1226(2011)$.

5. Yifan, D., Hao, H., Xiaoqiang, P., Jianmin, W. \& Feng, S. Research on error control and compensation in magnetorheological finishing. Appl. Opt. 50, 3321-3329 (2011).

6. Peng, X., Jiao, F., Chen, H., Tie, G. \& Feng, S. Novel magnetorheological figuring of KDP crystal. Chin. Opt. Lett. 9(10), 102201-1-102201-5 (2011).

7. Schinhaerl, M. et al. Utilisation of time-variant influence functions in the computer controlled polishing. Precis. Eng. 32, 47-54 (2008).

8. H. M. Martin et al., Polishing of a 6.5-m f/1.25 mirror for the first Magellan telescope. Proceedings of SPIE - The International Society for Optical Engineering, 47-55 (1999).

9. Zhou, L., Dai, Y. F., Xie, X. H., Jiao, C. J. \& Sheng-Yi, L. I. Model and Method to Determine Dwell Time in Ion Beam Figuring. Nanotechnol. Precis. Eng. 5, 107-112 (2007).

10. Li., Z. Z. et al. Removal of single point diamond-turning marks by abrasive jet polishing. Appl. Opt. 50, 2458-2463 (2011).

11. Zhou, L., Xu, M., Peng, W., Xie, X. \& Dai, Y. Fabrication of continuous phase plates with small structures based on recursive frequency filtered ion beam figuring. Opt. Express 25, 10765 (2017).

12. Su, X. et al. Fabrication of continuous phase plate using atmospheric pressure plasma processing. The International Journal of Advanced Manufacturing Technology. (2019).

13. Burge, J. H. et al. Development of optimal grinding and polishing tools for aspheric surfaces. Proc. SPIE - Int. Soc. Optical Eng. 4451, 153-164 (2001).

14. Kim, D. W., Martin, H. M. \& Burge, J. H. Control of Mid-spatial-frequency Errors for Large Steep Aspheric Surfaces. Imaging and Applied Optics Technical Digest (2012).

15. Kim, D. W., Martin, H. M. \& Burge, J. H. Calibration and optimization of computer-controlled optical surfacing for large optics. Proc. SPIE 8126, 15-1 (2011).

16. Kim, D. W., Sug-Whan, K. \& Burge, J. H. Non-sequential optimization technique for a computer controlled optical surfacing process using multiple tool influence functions. Opt. Express 17(24), 21850-21866 (2009).

17. Kim, D. W., Martin, H. M. \& Burge, J. H. Rigid conformal polishing tool using non-linear visco-elastic effect. Opt. Express 18(3), $2242-2257$ (2010).

18. Dunn, C. R. \& Walker, D. D. Pseudo-random tool paths for CNC sub-aperture polishing and other applications. Opt. Express 16, 18942-18949 (2008).

19. Dai, Y. F., Shi, F., Peng, X. Q. \& Li, S. Y. Restraint of mid-spatial frequency error in magneto-rheological finishing (MRF) process by maximum entropy method. Sci. China 52, 3092-3097 (2009).

20. Hon-Yuen, T., Haobo, C. \& Zhichao, D. Peano-like paths for subaperture polishing of optical aspherical surfaces. Appl. Opt. 52(15), 3624-3636 (2013).

21. Nie, X., Li, S., Shi, F. \& Hu, H. Generalized numerical pressure distribution model for smoothing polishing of irregular midspatial frequency errors. APPL. OPT. 6(53), 1020-1027 (2014).

22. Wang, D. Von Mises Stress in Chemical-Mechanical Polishing Processes. J. Electrochem. Soc. 144, 1121 (1997).

23. Rao, S. S. The Finite Element Method in Engineering (Pergamon, 1989).

24. Preston, F. W. The theory and design of plate glass polishing machines. J. Soc. Glas. Technol. 11, 214-256 (1927).

25. Yong, S., Dae Wook, K., Martin, H. M. \& Burge, J. H. Correlation-based smoothing model for optical polishing. Opt. Express 21, 28771-28782 (2013).

26. Tuell, M. T. "Novel tooling for production of aspheric surfaces," M.S (2002).

\section{Acknowledgements}

The financial support for this research is the National Natural Science Foundation of China (NSFC) (No. 51835013, No. 51775551) and the National Science joint Foundation of China (U180120098).

\section{Author contributions}

Conceptualization, C.S. and X.Q.N.; Methodology, X.Q.N.; Software, X.Q.N.; Validation, X.Q.N., Z.F.L. and F.S.; Formal analysis, F.S.; Investigation, W.L.Z.; resources, Z.F.L.; Writing—original draft preparation, X.Q.N.; Writing-review and editing, W.L.Z.; Visualization, W.L.Z.; Supervision, F.S.; Project administration, F.S.; Funding acquisition, F.S. and C.S.

\section{Competing interests}

The authors declare no competing interests.

\section{Additional information}

Correspondence and requests for materials should be addressed to W.Z.

Reprints and permissions information is available at www.nature.com/reprints.

Publisher's note Springer Nature remains neutral with regard to jurisdictional claims in published maps and institutional affiliations.

\begin{abstract}
(c) (i) Open Access This article is licensed under a Creative Commons Attribution 4.0 International cc. License, which permits use, sharing, adaptation, distribution and reproduction in any medium or format, as long as you give appropriate credit to the original author(s) and the source, provide a link to the Creative Commons license, and indicate if changes were made. The images or other third party material in this article are included in the article's Creative Commons license, unless indicated otherwise in a credit line to the material. If material is not included in the article's Creative Commons license and your intended use is not permitted by statutory regulation or exceeds the permitted use, you will need to obtain permission directly from the copyright holder. To view a copy of this license, visit http://creativecommons.org/licenses/by/4.0/.
\end{abstract}

(C) The Author(s) 2020 